\title{
CRIANÇAS ACOMETIDAS COM COVID-19 NO ESTADO DO CEARÁ: UM ESTUDO EPIDEMIOLÓGICO
}

\author{
CHILDREN AFFECTED WITH COVID-19 IN THE STATE OF CEARÁ: \\ AN EPIDEMIOLOGICAL STUDY
}

\author{
Lívia Evlin Felix Brandão ${ }^{1}$ \\ Yuri Charllub Pereira Bezerra ${ }^{2}$ \\ Renata Lívia Silva Fonseca Moreira de Medeiros ${ }^{3}$ \\ Geane Silva Oliveira ${ }^{4}$
}

RESUMO: Objetivo: Traçar o perfil epidemiológico das crianças acometidas com a COVID-19 no estado do Ceará. Metodologia: Trata-se de um estudo documental, descritivo, de natureza quantitativa, tendo a população composta por todos os dados fornecidos pela plataforma do IntegraSUS, uma plataforma de transparência da gestão pública de saúde do Ceará, obtidos no período de julho de 2021 a setembro de 2021. A amostra foi composta por todos os dados presente na plataforma que corresponderam aos seguintes critérios de inclusão: Indicadores do coronavírushistórico de internações, óbitos, e acompanhamento de testes de COVID-19, acompanhamento dos atendimentos por síndrome gripal, positividade de exames, regulação estadual pacientes COVID-19. Os dados obtidos foram tabulados, apresentados em forma de gráficos e tabelas e analisados por meio da estatística descritiva, simples, conforme as variáveis provenientes do roteiro semiestruturado e, em seguida, correlacionados e discutidos através do cunho científico e seguindo os princípios éticos e legais da Resolução 466/12. Resultados: Foi possível identificar que o maior índice de casos confirmados foram na faixa etária de 10 a 14 anos e em relação ao gênero no sexo feminino, porém foi possível identificar que o maior risco não está nessas crianças e sim, nas que têm idade entre 00 a 04 anos, pois possuem uma taxa de letalidade e óbito maior e, em relação ao gênero, são as crianças do sexo masculino que estão mais propícias a ter o quadro grave da doença. Conclusão: Diante disso, conclui-se que, apesar das crianças estarem em um grupo de menor risco, elas não se isentam dos riscos e gravidades da doença, além de serem veículo de transmissão da doença, fazendo-se necessário todas as medidas preventivas e de enfrentamento da COVID-19 com essa população.

\footnotetext{
${ }^{1}$ Acadêmicos de Enfermagem da Faculdade Santa Maria (FSM), Cajazeiras-PB.

${ }^{2}$ Docente do curso de Enfermagem da Faculdade Santa Maria (FSM), Cajazeiras-PB.

${ }^{3}$ Docente do curso de Enfermagem da Faculdade Santa Maria (FSM), Cajazeiras-PB.

${ }^{4}$ Docente do curso de Enfermagem da Faculdade Santa Maria (FSM), Cajazeiras-PB.
} 
Palavras chave: COVID-19; Crianças; Infecção por Coronavírus; Epidemiologia.

ABSTRACT: Objective: To trace the epidemiological profile of children affected by COVID-19 in the state of Ceará. Methodology: This is a documentary, descriptive study of a quantitative nature, with the population comprising all data provided by the IntegraSUS platform, a transparency platform for public health management in Ceará, obtained from July 2021 to September 2021. The sample consisted of all data present on the platform that met the following inclusion criteria: Coronavirus indicators - history of hospitalizations, deaths, and follow-up of COVID-19 tests, follow-up of care for flu syndrome, positivity of exams, state regulation COVID-19 patients. The data obtained were tabulated, presented in the form of graphs and tables and analyzed using simple descriptive statistics, according to the variables from the semi-structured script, and then correlated and discussed using a scientific approach and following the ethical and legal principles of the Resolution 466/12. Results: It was possible to identify that the highest rate of confirmed cases were in the age group of 10 to 14 years and in relation to gender in females, but it was possible to identify that the greatest risk is not in these children, but in those aged between 00 a 04 because they have a higher lethality and death rate, and in relation to gender, male children are more likely to have the serious condition of the disease. Conclusion: In view of this, it is concluded that despite the children being in a lower risk group, they are not exempt from the risks and seriousness of the disease, in addition to being a vehicle for disease transmission, requiring all preventive and coping measures by COVID -19 with this population. 


\section{INTRODUÇÃO}

A COVID-19, nome atribuído pela OMS (Organização mundial da Saúde), a doença causada por um novo coronavírus, o SARS-CoV-2, que está relacionado aos quadros de síndrome respiratória aguda grave, esse vírus foi identificado em 7 de janeiro de 2020, na cidade chinesa de Wuhan, após um surto de pneumonia. A doença tomou uma proporção rápida que resultou na pandemia, sendo classificada assim pela OMS em 11 de março de 2020 (TAVARES et al., 2020).

Logo foi comprovada a transmissão de pessoa para pessoa, a partir de secreções respiratórias, expelidas através da fala, tosse, espirros e a respiração, em alguns estudos, já é mencionado através das fezes, urina, bem como, através da transmissão vertical. Apresenta potencial contaminante tanto pessoas sintomáticas como assintomáticas, e as pessoas que a doença se encontra em estágio de incubação (RAMOS et al., 2020).

Segundo a Organização Pan-Americana da Saúde (OPAS-2020), muitas pessoas conseguem se recuperar sem atendimento hospitalar, em contrapartida, as que precisam de atendimento hospitalar desenvolvem bastante dificuldade de respirar. As pessoas idosas e pessoas com comorbidades têm maior risco de ficarem gravemente doentes, isso não anula o fato de pessoas jovens e crianças apresentarem quadro grave da doença.

De acordo com a Sociedade Brasileira de Pediatria (2020), as crianças, apesar de serem menos vulneráveis à doença e, muitas vezes, apresentarem casos leves, podem apresentar infecção assintomática, contribuindo para infecção do COVID-19 e, em casos graves, podem ter um desconforto respiratório agudo. Os sintomas mais presentes nas crianças são: coriza, garganta inflamada, tosse seca e congestão nasal.

Segundo o Instituto Nacional de Saúde da Mulher, da Criança e do Adolescente Fernandes Figueira da Fundação Oswaldo Cruz (IFF/Fiocruz 2020), mesmo sabendo que as crianças e adolescentes apresentam um risco menor da 
doença, a pandemia de COVID-19 apresenta algumas consequências sobre a saúde de crianças e adolescentes no Brasil, sendo capaz de apresentar potencial bastante negativo em relação ao que apresenta os países da Europa e América do Norte.

Devem ser considerados alguns fatores sob o risco de aumento na morbimortalidade, são eles: a composição demográfica da população brasileira, com alto número de crianças e adolescentes; contingente de crianças com condições crônicas com controle insuficiente; desafios no acesso e qualidade do cuidado na Atenção Primária à Saúde; desafios no acesso e qualidade do cuidado pediátrico de maior complexidade, visando grande pressão no sistema hospitalar, gerando, inclusive, a desativação de leitos pediátricos e o aumento da vulnerabilidade social (IFF/Fiocruz, 2020).

Segundo a revista "Época", o Ceará, que é uma das 27 unidades federativas do Brasil e está situado no norte da Região Nordeste, nos primeiros meses de 2020, contabilizou 11.785 casos de crianças e adolescentes até 19 anos diagnosticados com coronavírus, e as internações não pararam de crescer. Diante disso, esse estudo teve como objetivo traçar o perfil epidemiológico das crianças acometidas com COVID-19, no referido estado.

A presente pesquisa se justifica com base no atual cenário da saúde, e no desejo de ampliar o conhecimento científico, trazendo informações importantes e específicas sobre o perfil epidemiológico das crianças com COVID, no estado do Ceará. A mesma é de grande relevância na atualidade, por se tratar de uma doença recente e que está em fase de construção bibliográfica, contribuindo, assim, tanto para o público acadêmico como para os profissionais, podendo gerar melhorias assistenciais e no cuidado ao paciente.

\section{METODOLOGIA}

A presente pesquisa trata-se de um estudo epidemiológico, descritivo, de natureza quantitativa, onde reuniu dados epidemiológicos para realizar a pesquisa. O mesmo é uma pesquisa documental realizada a partir de dados do IntegraSUS, 
sendo intitulado Crianças acometidas com COVID-19 no estado do Ceará: Um estudo epidemiológico.

Para Gil (2017), a pesquisa documental é semelhante à pesquisa bibliográfica, tendo como principal diferença as fontes onde os dados são coletados. $\mathrm{Na}$ pesquisa documental possui duas categorias, as que não receberam nenhum tratamento analítico, que apresenta os documentos conservados em arquivos de órgãos públicos e instituições privadas, tais como, associações científicas, igrejas, sindicatos, partidos políticos etc. Que inclui outros documentos como cartas pessoais, diários, fotografias, gravações, memorandos, regulamentos, ofícios, boletins etc. De outro lado, há os documentos que de alguma forma já foram analisados, são eles: relatórios de pesquisa, relatórios de empresas, tabelas estatísticas etc.

Para este estudo foram realizadas 7 etapas: 1) determinação dos objetivos; 2) elaboração do plano de trabalho; 3) identificação das fontes; 4) localização das fontes e obtenção do material; 5) tratamento dos dados; 6) confecção das fichas e redação do trabalho; 7) construção lógica e redação do trabalho. A depender do tipo de documento utilizado para realização da coleta de dados, não necessariamente, a pesquisa irá se desenvolver de acordo com as etapas expostas acima (GIL, 2017).

A abordagem quantitativa consiste em tudo que pode ser quantificável, ou seja, tudo o que for concreto, com o intuito de investigar características de fatos, aspectos e fenômenos, analisando-os e traduzindo números em ideias e informações. Utiliza-se de recursos formais, como técnicas estatísticas, caracterizadas pela precisão e controle dos dados coletados e analisados (LAKATOS; MARCONI, 2017).

O presente estudo teve como cenário o Estado do Ceará, que está localizado na região Nordeste do Brasil. O estado surgiu através da doação da Capitania Siará, em 1535, e sua fundação ocorreu em 1603, possuindo 184 municípios, sua população corresponde a 8,4 milhões de habitantes, segundo o IBGE. No referido estado, foram confirmados 721.495 casos de COVID-19 até 01/05/2021, cuja taxa de mortalidade acumulada (2020 e 2021) é de 208,5 por 100 mil habitantes, representando uma letalidade de 2,6\%. (SEVIR, 2021). 
Essa pesquisa teve a população composta por todos os dados fornecidos pelo site do IntegraSUS, uma plataforma de transparência da gestão pública de saúde do Ceará, obtidos no período de julho de 2021 a setembro de 2021, através do índice de transparência do COVID-19, servindo para comprovação e levantamento de dados.

Essa plataforma tem por intuito integrar em tempo real as informações em saúde do estado do Ceará para acesso público e desenvolvimento de estratégias para tomada de decisão.

A amostra foi composta por todos os dados que corresponderem aos seguintes critérios de inclusão: Indicadores do coronavírus- histórico de internações, óbitos, e acompanhamento de testes de COVID-19, acompanhamento dos atendimentos por síndrome gripal, positividade de exames, regulação estadual pacientes COVID-19.

Para a coleta de dados, foi utilizado um roteiro semiestruturado composto por informações extraídas do IntegraSUS. Este tipo de roteiro trata-se de um conjunto de questionamentos sobre uma determinada temática com aporte teórico e em consonância com objetivos e hipóteses da investigação, previamente elaborados (OLIVEIRA, 2011). Os dados foram coletados nos meses de julho a setembro de 2021.

As informações adquiridas a partir deste estudo foram tabuladas, gerados os resultados, e apresentados em forma de gráficos e tabelas, sendo analisados por meio da estatística descritiva simples, conforme as variáveis provenientes do roteiro semiestruturado e, em seguida, correlacionados e discutidos através do cunho científico.

Por ser uma base de dados de domínio público, e por não se tratar de uma pesquisa que envolva diretamente seres humanos, não foi necessária a submissão do projeto ao Comitê de Ética e Pesquisa. Ressalta-se, porém, que foram preservados durante toda a pesquisa os preceitos éticos e legais, acompanhados dos referenciais básicos da bioética que são: a autonomia, não maleficência, beneficência e justiça. 


\section{RESULTADOS}

O Ceará foi o primeiro Estado do Nordeste a notificar casos de COVID-19. Foram notificados 2.485 .075 casos e foram confirmados positivos para COVID-19 944.287 casos até o momento. Sendo os casos confirmados 150.061 são crianças entre 00 a 14 anos.

Diante disso, foi coletado os dados em relação às crianças com COVID-19, no estado do Ceará. No gráfico 1, temos dados relacionados aos casos que apresentaram síndrome gripal:

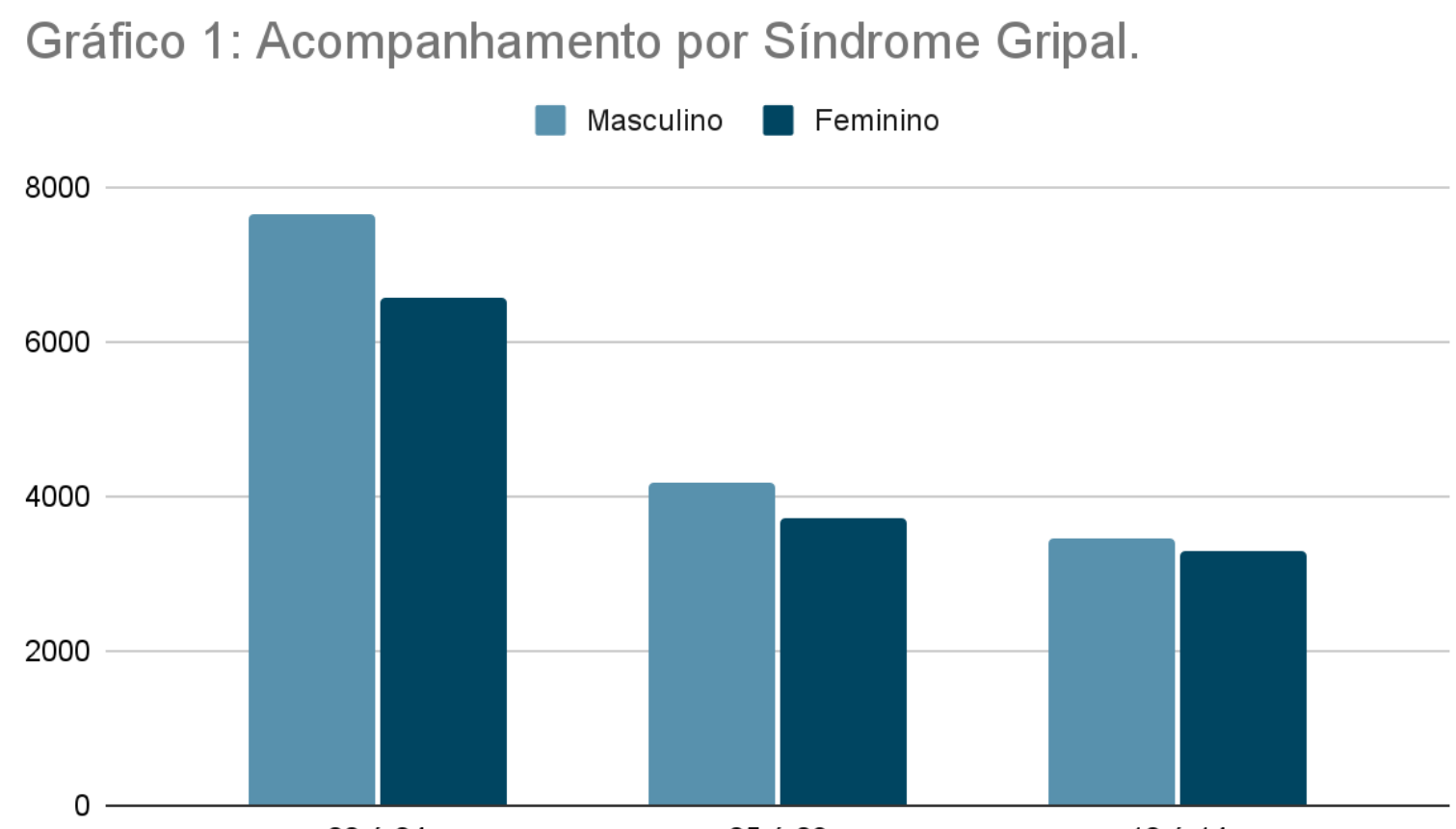

00 á 04

05 á 09

10 á 14

Fonte: Dados da pesquisa, 2021;

A COVID-19, no seus sintomas, está diretamente ligado a síndrome gripal, fazendo com que pessoas que apresentam esse diagnóstico realizam a testagem para COVID-19. Sendo assim, foi realizado um acompanhamento com o público que apresentava síndrome gripal, sendo possível identificar que crianças de 00 a 04 anos do sexo masculino foram 7.656 casos e do sexo feminino 6.561 casos; de 05 a 
09 anos do sexo masculino foram 4.172 casos e do sexo feminino foram 3.708 casos; na idade de 10 a 14 anos do sexo masculino foram 3.460 casos e no sexo feminino foram 3.279 casos.

\section{Gráfico 2: Casos Confirmados de crianças com COVID-19 no Estado do Ceará}

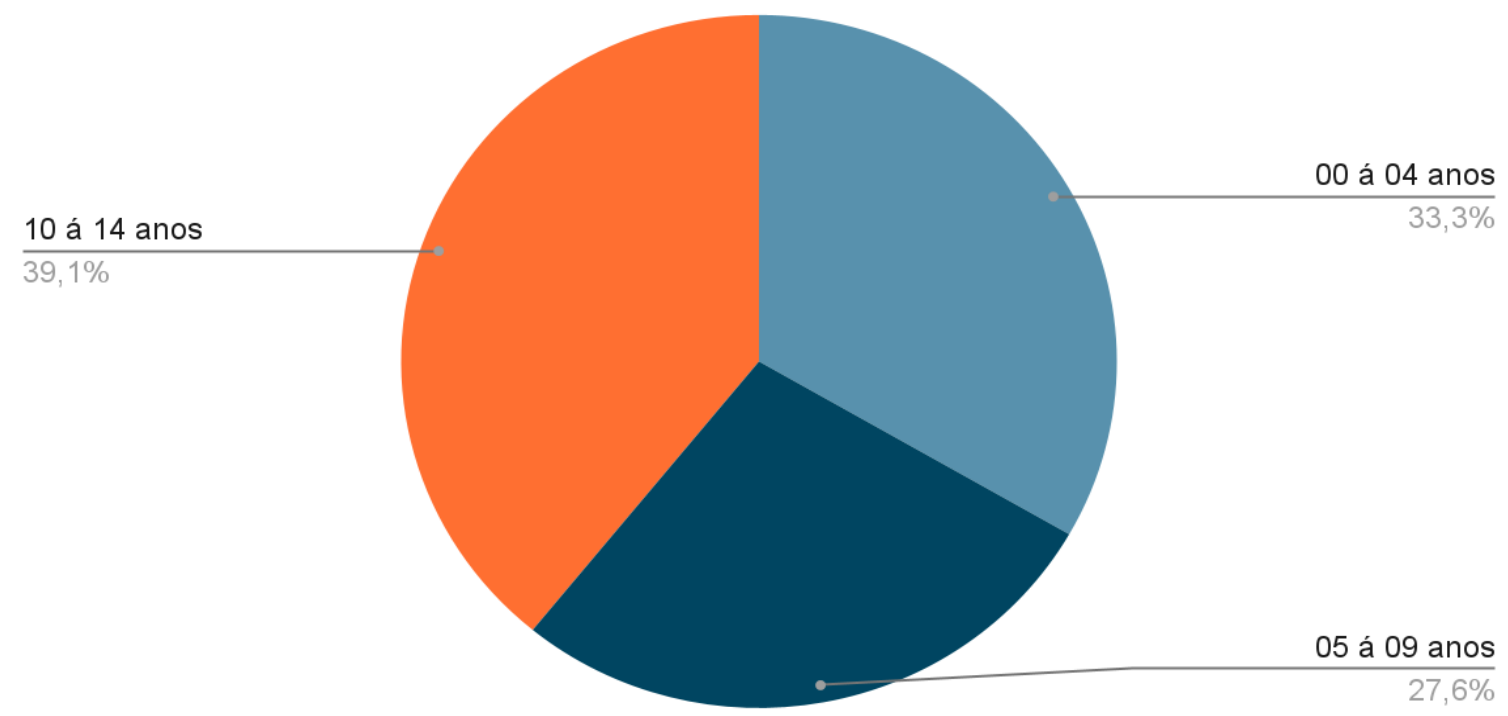

Fonte: Dados da pesquisa, 2021;

Nesse item, foi possível identificar que entre 00 a 04 anos foram confirmados 10.162 casos, no sexo feminino, e 10.662, no sexo masculino, totalizando, assim, $33,3 \%$ dos casos confirmados em crianças, na faixa etária de 05 a 09 anos foram confirmados 8.417 casos no sexo feminino, e 8.423 no sexo masculino, totalizando, assim, 27,6\% dos casos e, na faixa etária de 10 a 14 anos foram confirmados 11.931 casos, no sexo feminino, e 11.119 casos, no sexo masculino, totalizando $39,1 \%$ dos casos. Com isso, foi possível identificar que, no Estado do Ceará, o índice de casos confirmados foi maior na faixa etária de 10 a 14 anos, além disso, verificou-se uma maior proporção do sexo feminino.

Em seguida, no gráfico 3, está relacionada a taxa de letalidade do COVID-19 em crianças no Estado do Ceará: 


\section{Gráfico 3: Taxa de Letalidade das Crianças com COVID-19 no Estado do Ceará.}

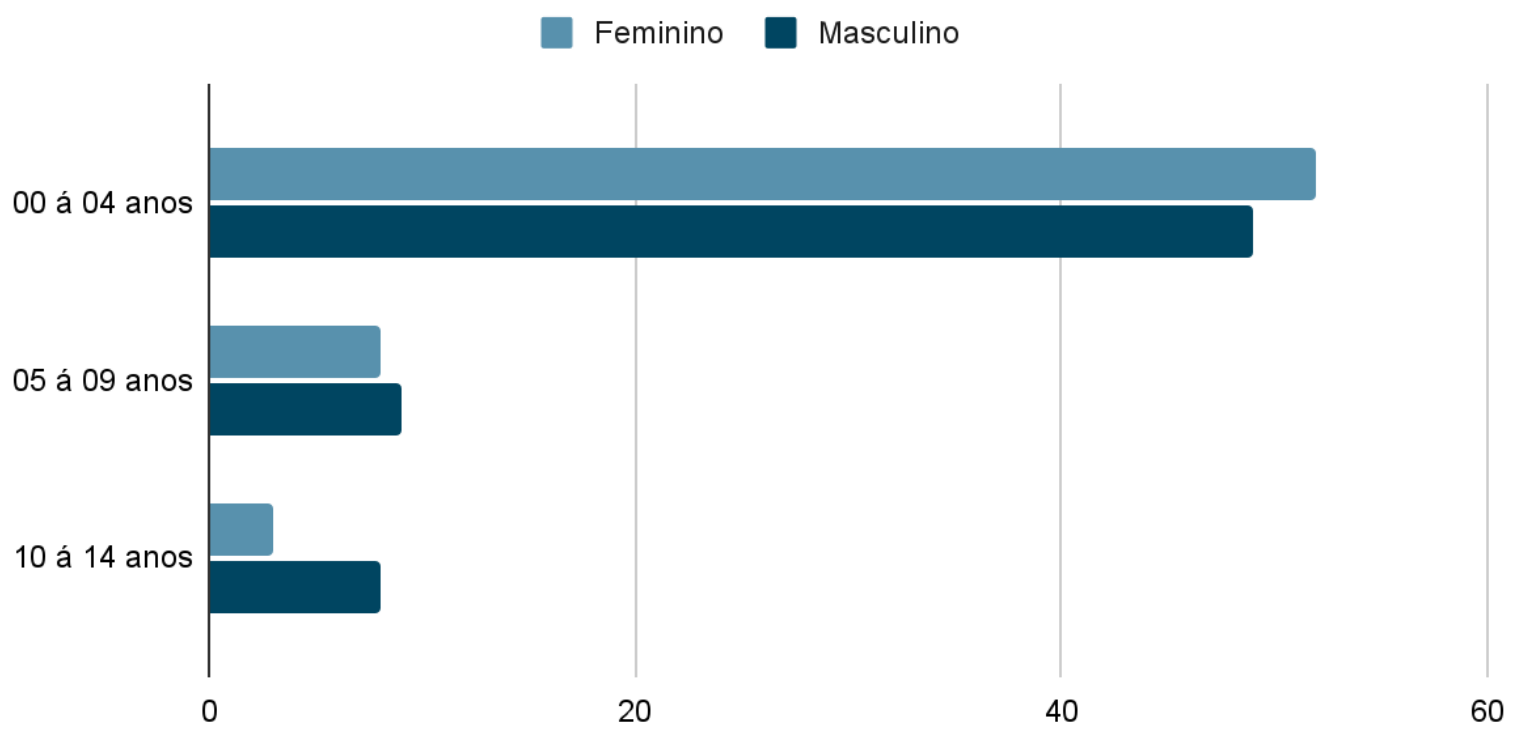

Fonte: Dados da pesquisa, 2021;

De acordo com esse item, foi possível identificar que na faixa etária de 00 a 04 anos o índice de letalidade em crianças do sexo feminino foi de $0,56 \%$ e, no sexo masculino, 0,49\%, correspondendo, assim, 0,52\% ao índice em relação as faixas etárias gerais, na idades correspondentes entre 05 a 09 anos, no sexo feminino o índice é de $0,08 \%$ e, no sexo masculino, de $0,09 \%$ e, em relação às faixas etárias, em geral, corresponde a $0,09 \%$, as idades de 10 a 14 anos correspondem, no sexo feminino, a $0,03 \%$ dos casos e, no sexo masculino, a $0,08 \%$ e em relação às faixas etárias em geral corresponde a $0,06 \%$. Sendo assim, a faixa etária que apresentou mais índice de letalidade foi entre 00 a 04.

No gráfico 4, está relacionado o Histórico de Internação por COVID-19 nas crianças do Estado do Ceará.

Nesse item, foi possível identificar que foram internados na UTI Neonatal 17,24\%, na UTI Infantil 64,91\%, na enfermaria neonatal $40 \%$ e na enfermaria infantil $72,53 \%$. Sendo assim, é possível identificar que os setores que tiveram mais atendimento foi a UTI Infantil e a Enfermaria Infantil. 


\section{Gráfico 4: Histórico de internação COVID-19 nas crianças do Estado do Ceará \\ Porcentagem de pacientes internos}

80

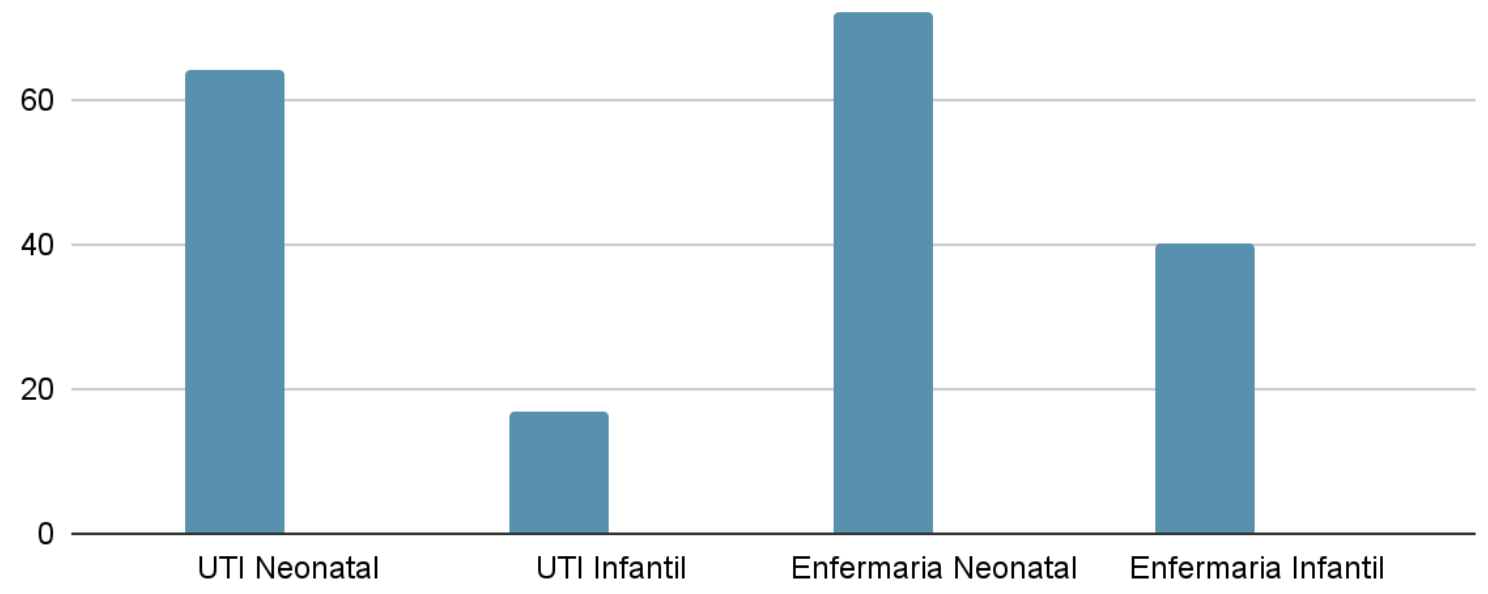

Fonte: Dados da pesquisa, 2021;

O Gráfico 5 é referente aos óbitos por COVID-19 em crianças do Estado do Ceará.

Nesse item, foi possível identificar que na faixa etária de 00 a 04 anos, no sexo feminino, foram 51 óbitos e, no masculino, foram 52 óbitos; na faixa etária de 05 a 09 foram, no sexo feminino, 07 óbitos e, no sexo masculino, 08 óbitos; na faixa etária de 10 a 14 anos foram, no sexo feminino, 4 óbitos e, no sexo masculino, 9 óbitos. Diante desses dados, é possível identificar que a taxa maior de óbito foi na faixa etária de 00 a 04 anos e, quanto ao gênero, a maior taxa de óbito foi no sexo masculino. 


\section{Gráfico 5: óbitos por COVID-19 em Crianças do Estado do Ceará.}

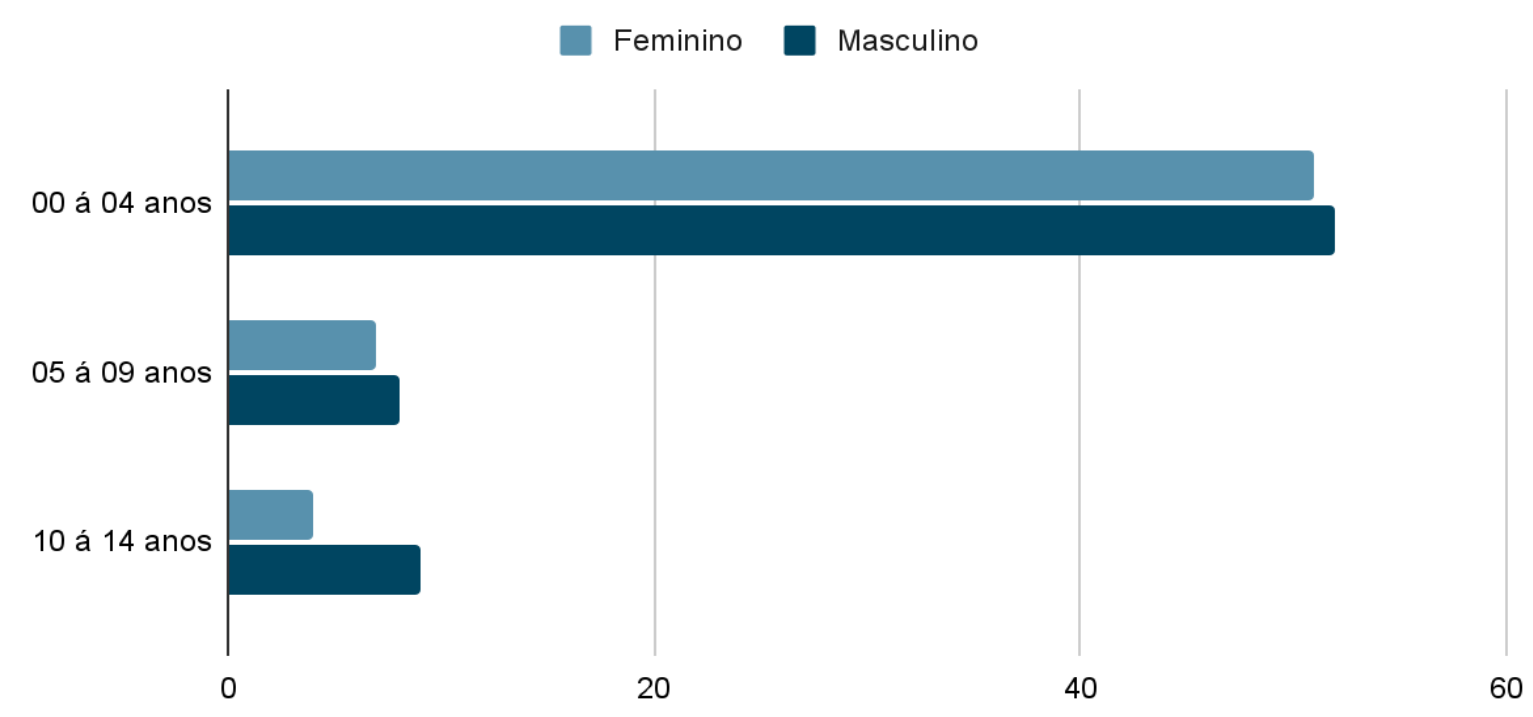

Fonte: Dados da pesquisa, 2021;

\section{DISCUSSÃO}

Neste estudo, avaliamos o perfil das crianças e adolescentes acometidos por COVID-19, no estado do Ceará, sendo esclarecido que esta população foi menos afetada e com uma evolução mais benéfica em relação aos adultos, confirmando, assim, achados de estudos anteriores. Isso pode estar relacionado a inúmeros fatores, sendo eles: as aulas das escolas que foram suspensas, diminuindo o contato entre as crianças e pessoas contaminadas; outras hipóteses apresentadas por estudos é que a maioria das crianças com COVID-19 são assintomáticas, sendo assim, não são diagnosticadas (SHE, 2020).

Outro problema é que as crianças possuem particularidades e não conseguem descrever de forma clara e objetiva os sintomas que estão sentindo ou até mesmo com quem teve contato, caso seja necessário uma investigação, o que acaba se tornando um enorme desafio para que se possa proteger, diagnosticar e 
cuidar dessa população. Compreende-se, também, que essa população convive com seus familiares de forma agrupada, ou seja, mesmo que pra eles geralmente a infecção aconteça de forma leve, eles podem acabar transmitindo a doença para seus familiares que, na maioria das vezes, acabam tendo os sintomas graves da doença (HILLESHEIM et al., 2020).

Nesse cenário caótico de pandemia, as crianças e adolescentes não foram poupados da infecção por COVID-19. Essa faixa etária apresenta de $1 \%$ a 5\% dos casos confirmados, porém são casos mais leves que os adultos, e as mortes são raras. Entretanto, esse grupo pode ter casos de infecção assintomática, que mostram que a eliminação do vírus, tanto nas secreções respiratórias como nas fezes, acontece de forma mais longa que nos adultos, o que aumenta a taxa de disseminação da doença, aumentando o número de infectados (GÓES et al., 2020).

A COVID-19, no seus sintomas, está diretamente ligada à síndrome gripal, fazendo com que pessoas que apresentam esse diagnóstico realizem a testagem para COVID-19. No Ceará, a faixa etária que mais apresentou síndrome gripal foi crianças de 00 a 04 anos. Sabendo que a maioria dessa população apresenta casos leves e, de forma geral, as síndromes gripais, destacamos que o acompanhamento, caso seja necessário, será feito pela atenção primária em saúde, onde serão empregadas medidas de precaução domiciliar com o objetivo de conter a disseminação da doença para outras pessoas (SAFADI, 2020).

A Sociedade Brasileira de Pediatria destaca a importância de seguir as medidas de prevenção para conter a transmissão da COVID-19, entre os profissionais de saúde que estão na linha de frente desses serviços pediátricos, como é o caso da atenção primária. Os profissionais desses serviços são responsáveis por ceder todas as informações necessárias aos cuidadores dessas crianças para que todas as dúvidas sejam sanadas de modo que contribua para conter a disseminação da COVID-19 (SAFADI, 2020).

Nos casos confirmados, no estado do Ceará, foi possível identificar que na idade de 10 a 14 anos está o maior índice de contaminação, acompanhado disso, encontra-se o sexo feminino, divergindo do contexto brasileiro que apresenta maior frequência de acometimento em crianças do sexo masculino, e a mediana se 
encontra em crianças de 6 anos. Porém os óbitos são mais recorrentes na idade de 00 a 04, e de maior índice no sexo masculino.

Embora os sintomas principais sejam os mesmos que nos adultos, vemos uma diferença bem grande na evolução dos casos. De acordo com a literatura, a gravidade do quadro clínico foi nitidamente maior entre as crianças menores de 3 anos de idade, com frequência de hospitalização e necessidade de UTI superior à das crianças maiores (RABHA et al., 2020). No presente estudo, foi possível identificar que as UTI's e enfermaria infantil, apresentavam uma ocupação maior dos leitos, e também foi possível identificar que a letalidade maior está na idade de 00 a 04 anos.

A análise desses dados epidemiológicos, perfil dos óbitos e letalidade é fundamental para a construção de medidas preventivas e de enfrentamento da COVID-19 no Brasil, especialmente, com essa população infanto-juvenil, pois mesmo esta não sendo a população mais afetada, trata-se de um grupo que, além de ser um veículo de transmissão da doença, apresenta uma letalidade pela doença considerável, visando seus níveis crescentes. Os resultados expostos demonstram a necessidade de atender às demandas desse público, especialmente de indivíduos menores de 4 anos de idade. Visando ser um grupo que, dentro do Brasil, ainda não entra na faixa etária para ser contemplado com os imunobiológicos existentes, os gestores devem adotar as medidas mais adequadas para o cuidado dessa população.

\section{CONCLUSÃO}

O presente estudo evidenciou que as crianças, no estado do Ceará, que tiveram COVID-19, além de serem transporte para doença, apresentaram quadros graves da doença, o que é preocupante, pois, diante dos dados expostos, foi possível identificar que o maior índice de casos confirmados foram na faixa etária de 10 a 14 anos e, em relação ao gênero, no sexo feminino. Porém foi possível identificar que o maior risco não está nessas crianças e sim, nas que tem idade entre 
00 a 04, pois possuem uma taxa de letalidade e óbito maior e, em relação ao gênero, são as crianças do sexo masculino que estão mais propícias a ter o quadro grave da doença.

Sendo assim, é necessário que as mesmas tenham os cuidados necessários quanto à lavagem das mãos, uso das máscaras, distanciamento social, principalmente por ser um grupo que está vulnerável quanto à imunização, pois, até o presente momento, só serão contempladas com a vacinação as crianças acima de 12 anos, no território brasileiro.

As limitações encontradas nesta pesquisa são referentes à temática ser bem atual, sem muitas informações relevantes e concretas com poucos estudos. Desse modo, sugere-se mais estudos com essa temática, ampliando, assim, o conhecimento nessa área, sendo possível entender mais sobre essa patologia e como ela vem a intervir nesse público.

\section{REFERÊNCIAS BIBLIOGRÁFICAS}

ALMEIDA, Carlos Alberto Nogueira; LAMOUNIER, Joel Alves. O pediatra como protagonista no enfrentamento à COVID-19 em 2021.

BE13-Boletim-do-COE - saude.gov.br - Ministério da Saúde Disponível em: https://portalarquivos.saude.gov.br > 2020.

BRASIL. Ministério da Saúde. Secretaria de Atenção à Saúde. Departamento de Ações Programáticas Estratégicas. Política Nacional de Atenção Integral à Saúde da Criança: orientações para implementação / Ministério da Saúde. Secretaria de Atenção à Saúde. Departamento de Ações Programáticas Estratégicas. - Brasília: Ministério da Saúde, 2018. 180 p.: il. ISBN 978-85-334-2596-5

Covid-19: aumento de casos em crianças dobra ocupação de UTIs no Ceará - Época (globo.com).

DONG Y, et al. Epidemiological characteristics of 2143 pediatric patients with 2019 coronavirus disease in China. Pediatrics. 2020.

FIOCRUZ. Instituto Nacional de Saúde da Mulher, da Criança e do Adolescente Fernandes Figueira. COVID-19 e Saúde da Criança e do Adolescente. Ago., 2020. Disponível em: < https://portaldeboaspraticas.iff.fiocruz.br/atencaocrianca/covid-19-saude-crianca-e-adolescente>

GIL, A. C. Como elaborar projetos de pesquisa. 6. ed. São Paulo: Editora Atlas, p. 192, 2017.

HILLESHEIM, Danúbia et al. Síndrome respiratória aguda grave por COVID-19 em crianças e adolescentes no Brasil: perfil dos óbitos e letalidade hospitalar até a 38a Semana Epidemiológica de 2020. Epidemiologia e Serviços de Saúde, v. 29, 2020. 
LAKATOS, E. M.; MARCONI, M. A. Fundamentos metodologia científica. 8.ed. São Paulo: Editora Atlas, 2017a. 368 p.

LUDVIGSSON JF. Systematic review of COVID-19 in children shows milder cases and a better prognosis than adults. Acta Paediatr. 2020.

PARK SE. Epidemiologia, virologia e características clínicas de síndrome respiratória aguda grave - coronavírus-2 (SARS-CoV-2; Doença coronavírus-19). Clin Exp Pediatr. 2020.

RAMOS, Regina Terse et al. Aspectos respiratórios da COVID-19 na infância: o que o pediatra precisa saber? Resid Pediatr, v. 10, n. 2, p. 1-15, 2020.

RIBEIRO, Keyse Mirelle Carregosa et al. MANIFESTAÇÕES CLíNICAS DO COVID-19 NA PEDIATRIA: REVISÃO DE LITERATURA. Semana de Pesquisa do Centro Universitário Tiradentes-SEMPESq-Alagoas, n. 8, 2020.

SAFADI, Marco Aurélio Palazzi. As características intrigantes da COVID-19 em crianças e seu impacto na pandemia. Jornal de Pediatria, v. 96, n. 3, p. 265-268, 2020.

SILVA, João Ricardo Azevedo et al. COVID-19 em Pediatria: um panorama entre incidência e mortalidade. Rev Residência Pediátrica. Sociedade Brasileira de Pediatria, v. 383, 2020.

SHEN K, YANG Y, WANG T, ZHAO D, JIANG Y, JIN Y, et al. Diagnosis, treatment, and prevention of 2019 novel coronavirus infection in children: experts' consensus statement. World J Pediatr. 2020 Jun;16(3):223-31.

SHE J, LIU L, LIU W. COVID-19 epidemic: Disease characteristics in children. J MedVirol. 2020.

TAVARES, M, Silva, C. Da emergência de um novo vírus humano à disseminação global de uma nova doença. Capítulo I: Epidemiologia da COVID-19_26-02-2020 [consultado abril 2021;].

WHYTE CS, MORROW GB, MITCHELL JL, CHOWDARY P, MUTCH NJ. Fibrinolytic abnormalities in acute respiratory distresssyndrome (ARDS) and versatility of thrombolytic drugs to treat COVID-19. Journal of Thrombosis and Haemostasis; 2020. 